\title{
FUNDAMENTALS OF EQUILIBRIUM PROBLEMS
}

\author{
MUHAMMAD ASLAM NOOR
}

\begin{abstract}
Equilibrium problems theory provides us with a unified, natural, innovative and general framework for studying a wide class of linear and nonlinear problems arising in finance, economics, image reconstructions, medical imaging, ecology, network analysis, transportation. elasticity, operations research and optimization. In this work, we consider some new classes of equilibrium problems in the framework of convexity, invexity, $g$-convexity and prox-regular convexity. We also study a class of equilibrium problems involving the nondifferentability Lipschitz continuous functions, which is known as the hemiequilibrium problems. The auxiliary principle technique is used to suggest and analyze several iterative schemes for solving these classes of equilibrium problems. We consider the convergence analysis of these iterative algorithms under some mild conditions. We also introduce the concept of well-posedness for the equilibrium problems and obtain some interesting results. As special cases, we obtain several known and new results for variational inequalities and related optimization problems. Results obtained in this paper can be viewed as a nice and novel applications of the auxiliary principle technique in this fast growing and fascinating field.
\end{abstract}

Mathematics subject classification (2000): 91B50, 91B52,49J40, 90C26, 90C30.

Key words and phrases: equilibrium problems, variational inequalities, auxiliary principle, proximal methods, convergence, well-posedness.

\section{REFERENCES}

[1] E. Blum, W. Oettli, From Optimization and Variational Inequalities to Equilibrium Problems, Mathematics Students, 63, (1994), 123-145.

[2] F. H. Clarke, Optimization and Nonsmooth Analysis, J. Wiley and Sons, NY, 1983.

[3] F. H. Clarke, Y. S. LedyaeV, R. J. Stern and P. R. Wolenski, Nonsmooth Analysis and Control Theory, Springer-Verlag, New York, NY, 1998.

[4] G. CRistescu, L. LuPSA, Non-Connected Convexities and Applications, Kluwer Academic Publishers, Dordrecht, Holland, 2002.

[5] V. F. Dem'yanov, G. E. Savroulakis, L. N. Ployakova and P. D. Panagiotopoulos, Quasidifferentiability and Nonsmooth Modelling in Mechanics, Engineering and Economics, Kluwer Academic Publishers, Dordrecht, 1996.

[6] F. FloRES-BAZAN, Existence Theorems for Generalized Noncoercive Equilibrium Problems: The Quasi-Convex Case, SIAM J. Optim. 11, (2000), 675-690.

[7] M. Funushima, Equivalent Differentiable Optimization Problems and Descent Methods for Asymmmetric Variational Inequality Problems, Math. Program., 53, (1992), 99-110.

[8] F. GianNessi, A. MAUger, Variational Inequalities and Network Equilibrium Problems, Plenum Press, New York, NY, 1995.

[9] F. Giannessi, A. MAugeri AND P. M. Pardalos, Equilibrium Problems: Nonsmooth Optimization and Variational Inequality Models, Kluwer Academics Publishers, Dordrecht, Holland, 2001.

[10] G. Glowinski, J. L. LiONS AND R. TREMOLIERES, Numerical Analysis of Variational Inequalities, North-Holland, Amsterdam, Holland, 1981.

[11] D. Goeleven, D. Mantague, Well-posed Hemivariational Inequalities, Numer. Funct. Anal. Optim., 16, (1995), 909-921.

[12] D. KinderLeHRER, G. STAMPACCHIA, An Introduction to Variational Inequalities and Their Applications, Academic Press, London, England, 1980. 
[13] M. A. Hanson, On Sufficiency of the Kuhn-Tucker Conditions, J. Math. Anal. Appl., 80, (1981), $545-550$.

[14] J. L. Lions, G. Stampacchia, Variational Inequalities, Comm. Pure Appl. Math., 20, (1967), 491-512.

[15] R. LuCChetTI, F. PATRONE, A Characterization of Tykhonov Well-Posedness for Minimum Problems with Applications to Variational Inequalities, Numer. Funct. Anal. Optim., 3, (1981), 461-478.

[16] R. LuCChetTI, F. PATRONE, Some Properties of Well-Posed Variational Inequalities Governed by Linear Operators, Numer. Funct. Anal. Optim., 5, (1982-83), 349-361.

[17] B. MARTINET, Regularisation D'inequations Variationelles par Approximations Successive, Rev. d'Aut. Inform. Rech. Oper., 3, (1970), 154-159.

[18] G. Masteroeni, Gap Functions for Equilibrium Problems, J. Global Optim., 27, (2003), 411-426.

[19] S. R. Mohan, S. K. NeOgY, On Invex Sets and Preinvex Functions, J. Math. Anal. Appl., 189, (1995), 901-908.

[20] U. Mosco, Implicit Variational Problems and Quasi Variational Inequalities, Lecture Notes in Mathematics, Springe Verlag, Berlin, 543, (1976), 83-156.

[21] Z. NANWIEWICZ, P. D. PANAGIOTOPOUlos, Mathematical Theory of Hemivariational Inequalities and Applications, Marcel Dekker, Boston, 1995.

[22] M. Aslam Noor, On Variational Inequalities, Ph. D. Thesis, Brunel University, London, U. K., 1975.

[23] M. Aslam Noor, Some Recent Advances in Variational Inequalities, Part 1: Basic Concepts, New Zealand J. Math. 26, (1997), 53-80.

[24] M. Aslam Noor, Some Recent Advances in Variational Inequalities, Part 2: Other Concepts, New Zealand J. Math., 26, (1977), 229-255.

[25] M. Aslam Noor, Mixed Quasi Invex Equilibrium Problems, Internat. J. Math. Math. Sci., 2004, (2004), 3057-3067.

[26] M. Aslam Noor, Multivalued General Equilibrium Problems, J. Math. Anal. Appl., 283, (2003), 140-149.

[27] M. Aslam Noor, General Variational Inequalities, Appl. Math. Letters, 1, (1988), 119-121.

[28] M. Aslam Noor, Mixed Quasi Variational Inequalities, Appl. Math. Comput., 146, (2003), 553-578.

[29] M. Aslam NoOR, Some Development in General Variational Inequalities, Appl. Math. Comput., 152, (2004), 199-277.

[30] M. ASLAm NoOR, Iterative Schemes for Nonconvex Variational Inequalities, J. Optim. Theory Appl., 121, (2004), 163-176.

[31] M. Aslam NooR, Auxiliary Principle Technique for Equilibrium Problems, J. Optim. Theory Appl., 122, (2004), 371-386.

[32] M. Aslam Noor, On a Class of Nonconvex Equilibrium Problems, Appl. Math. Comput., 157, (2004), 553-566.

[33] M. Aslam Noor, Invex Equilibrium Problems, J. Math. Anal. Appl., 302, (2005), 463-475.

[34] M. Aslam NooR, New Approximation Schemes for General Variational Inequalities, J. Math. Anal. Appl., 251, (2000), 217-229.

[35] M. Aslam Noor, Nonconvex Functions and Variational inequalities, J. Optim. Theory Appl., 87, (1995), 615-630.

[36] M. ASLAm NooR, Variational-like Inequalities, Optimization, 30, (1994), 323-330.

[37] M. Aslam Noor, Invex $\epsilon$-Equilibrium Problems with Trifunction, Int. J. Pure Appl. Math., 13, (2004), 123-136.

[38] M. Aslam Noor, Multivalued Equilibrium Problems with Trifunction, Aust. J. Math. Anal. Appl., 1, (2004), 1-11.

[39] M. Aslam Noor, Hemiequilibrium Problems, J. Appl. Math. Stoch. Anal., 2004, (2004), 235-244.

[40] M. Aslam Noor, Hemivariational Inequalities, J. Appl. Math. Comput., 17, (2005), 59-72.

[41] M. Aslam NooR, Some Algorithms for Hemiequilibrium Problems, J. Appl. Math. Comput., 19, (2005), 135-146.

[42] M. Aslam Noor, Fundamentals of Mixed Quasi Variational Inequalities, Int. J. Pure Appl. Math., 15, (2004), 137-258.

[43] M. Aslam NoOR, Hemivariational-like Inequalities, J. Comput. Appl. Math., 182, (2005), 316-326.

[44] M. Aslam Noor, On Generalized Preinvex Functions and Monotonicites, J. Inequal. Pure Appl. Math., 5, (4) (2004), 1-9, Article 110.

[45] M. Aslam Noor, K. InaYat Noor, On Equilibrium Problems, Appl. Math. E-Notes, 4, (2004), $125-132$.

[46] M. Aslam Noor, K. Inayat Noor, Regularized Hemivariational Inequalities, Nonl. Funct. Anal. Appl., 10, (2005), 495-506.

[47] M. Aslam NooR, W. Oettli, On General Nonlinear Complementarity Problems and Quasi-equilibria, Le Matem., 49, (1994), 313-331. 
[48] P. D. PANAGIOTOPOUlos, Nonconvex Energy Functions, Hemivariational Inequalities and Substationarity Principles, Acta Mach. , 42, (1983), 160-183.

[49] P. D. PANAgiotopoulos, Hemivariational inequalities, Applications to Mechanics and Engineering, Springer Verlag, Berlin, 1993.

[50] M. PATRIKSSON, Nonlinear Programming and Variational Inequality Problems: A Unified Approach, Kluwer Academic Publishers, Dordrecht, Holland, 1999.

[51] R. A. Poliquin, R. T. Rockafellar and L. Thibault, Local Differentiability of Distance Functions, Transactions of American Mathematical Society, 352, (2000), 5231-5249.

[52] R. T. Rockafellar, Monotone Operators and Proximal Point Algorithm, SIAM J. Control Optim., 14, (1976), 877-898.

[53] G. StAmPACCHIA, Formes Bilineaires Coercitives sur les Ensembles Convexes, Compt. Rend. l'Acad. Sciences, Paris, 258, (1964), 4413-4416.

[54] T. WeIR, B. Mond, Preinvex Functions in Multiobjective Optimization, J. Math. Anal. Appl., 136, (1988), 29-38.

[55] X. Q. YAng, G. Y. Chen, A Class of Nonconvex Functions and Pre-variational Inequalities, J. Math. Anal. Appl., 169, (1992), 359-373.

[56] D. L. ZHU, P. MARCOTTE, Co-coercivity and its Role in the Convergence of Iterative Schemes for Solving Variational inequalities, SIAM J. Optim., 6, (1996), 714-726. 